\title{
A Case Report on the Right Cervical Lymph Node Metastasis of Hepatocellular Carcinoma
}

Zheng Zhao ${ }^{1,2}$, Junhao Maia ${ }^{2}$, Xinrui Zhang ${ }^{3}$, Honghong Yan ${ }^{1,2}$, Xiaoqi Chen ${ }^{1,2}$ and Xuekui Liu ${ }^{1,2 *}$

${ }^{1}$ State Key Laboratory of Oncology in South China, Guangzhou, Guangdong, P.R. China

${ }^{2}$ Department of Head and Neck Surgery, Cancer Center of Sun Yat-Sen University, Guangzhou, Guangdong, P.R. China

${ }^{3}$ Department of Otorhinolaryngology, Guangzhou Otolaryngology-Head and Neck Surgery Hospital, P.R. China

\begin{abstract}
Metastasis to lymph nodes in patients with hepatocellular carcinoma (HCC) usually occurs in abdominal lymph nodes, which rarely observed to occur in cervical lymph node metastasis. This phenomenon in the clinic is easily neglected. We encountered a patient who underwent radical resection for primary liver cancer 1 year ago, accompanied by metastasis to only a single cervical lymph node. This case suggests that in the follow-up of patients who underwent surgical resection for HCC should be noticed the possibility of cervical lymph node metastasis.
\end{abstract}

Keywords: Hepatocellular carcinoma; Right Cervical lymph node; Metastasis; Therapy; Primary liver cancer

\section{Introduction}

Metastatic Hepatocellular Carcinoma (HCC) is one of the most common cancers worldwide. The overall prognosis of patients with metastatic HCC is poor. Lymph node metastasis in patients with HCC is closely related to a lower survival rate. Hepatocellular carcinoma (HCC) is the most common, malignant tumor of the liver. Hepatocellular carcinoma (HCC) commonly metastasizes to the lungs, abdominal lymph nodes, adrenal glands, or bones. Distant lymph node metastases are rare in hepatocellular carcinoma. Metastasis to lymph nodes in patients with hepatocellular carcinoma (HCC) is generally observed to occur in regional chains of involvement. Here, we present a case a patient who underwent radical resection for primary liver cancer 1 year ago, accompanied by metastasis to only a single cervical lymph node.

\section{Case Report}

A 52-year-old Chinese man with B-type liver cirrhosis was admitted for the lower limb weakness for a duration of 5 days in June 2016. Laboratory examinations revealed the serum hemoglobin concentration to be $15.4 \mathrm{~g} / \mathrm{dL}$; the total bilirubin level was $15.5 \mathrm{umol} / \mathrm{L}$; the direct bilirubin level was $5.3 \mathrm{umol} / \mathrm{L}$; the albumin level was $43.4 \mathrm{~g} / \mathrm{L}$; the serum creatinine level was $100 \mathrm{umol} / \mathrm{L}$; the serum concentration of alphafetoprotein (AFP) was $11.66 \mathrm{ng} / \mathrm{nL}$. HBV DNA quantification was $4.3 \times 10^{-5} \mathrm{IU} / \mathrm{ml}$. On enhanced magnetic resonance imaging (Figure 1), a mass approximately $92 \times 83 \mathrm{~mm}$ was noted in the hepatic S6/S7.

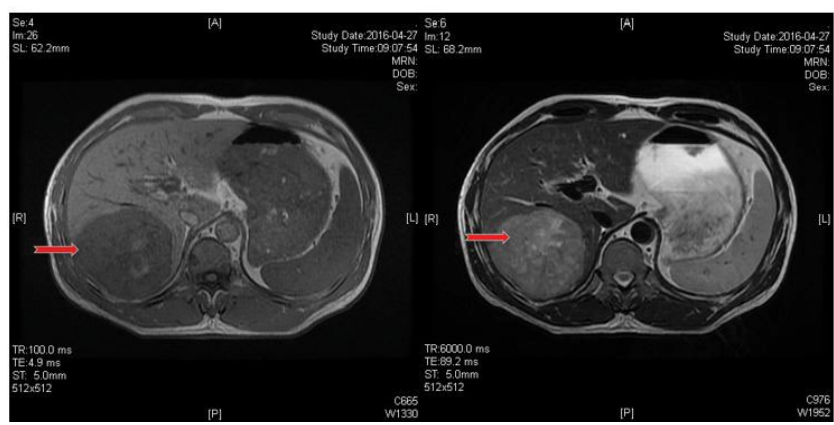

Figure 1: Magnetic resonance enhanced scan showing enlarged liver nodules (arrow).
Unfortunately, Unfortunately, patient did not perform PET/CT, neck ultrasonography and Computed Tomography [1-4]. We performed radical resection for primary liver cancer. The pathology revealed moderately differentiated HCC, trabecular type, with portal cancerous

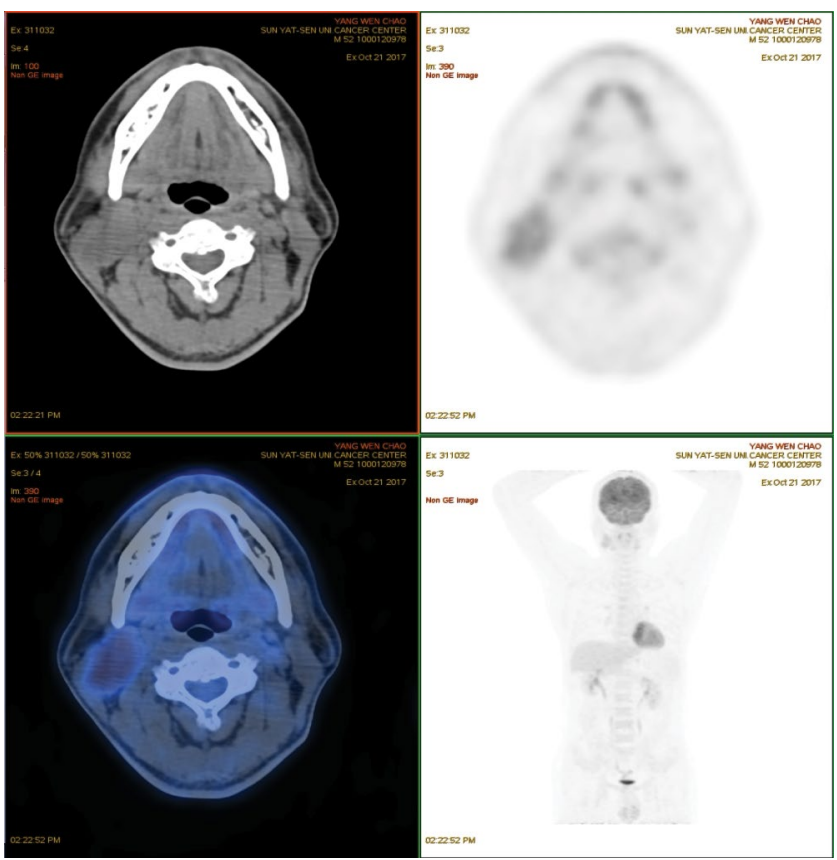

Figure 2: PET test showing uptakes of $18 \mathrm{~F}-\mathrm{FDG}$ into a right cervical lymph node.

*Corresponding author: Xue-kui Liu, PhD, Department of Head and Neck Surgery, Cancer Center, Sun Yat-Sen University, State Key Laboratory of Oncology in South China, Guangzhou, Guangdong 510060, P.R. China, Tel: +8602087343302; E-mail: liuxk@sysucc.org.cn

Received July 23, 2018; Accepted November 02, 2018; Published November 05, 2018

Citation: Zhao Z, Maia J, Zhang X, Yan H, Chen X, et al. (2018) A Case Report on the Right Cervical Lymph Node Metastasis of Hepatocellular Carcinoma. J Cancer Sci Ther 10: 329-331. doi: 10.4172/1948-5956.1000563

Copyright: (c) 2018 Zhao Z, et al. This is an open-access article distributed under the terms of the Creative Commons Attribution License, which permits unrestricted use, distribution, and reproduction in any medium, provided the original author and source are credited. 


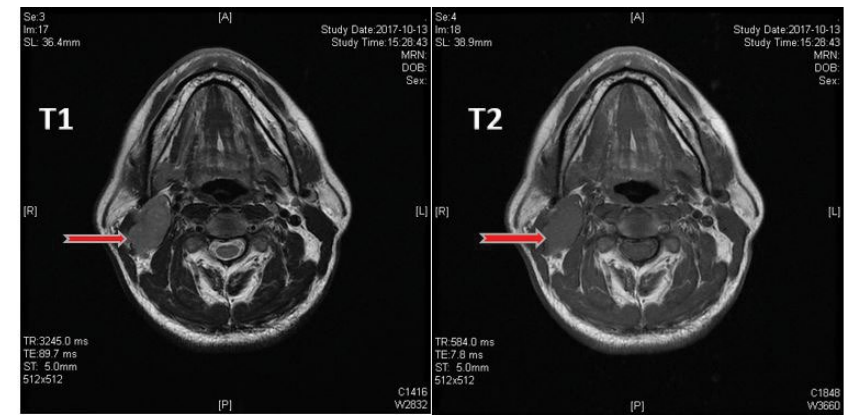

Figure 3: Magnetic resonance enhanced scan showing enlarged right cervica lymph node (arrow), which were easily observed.

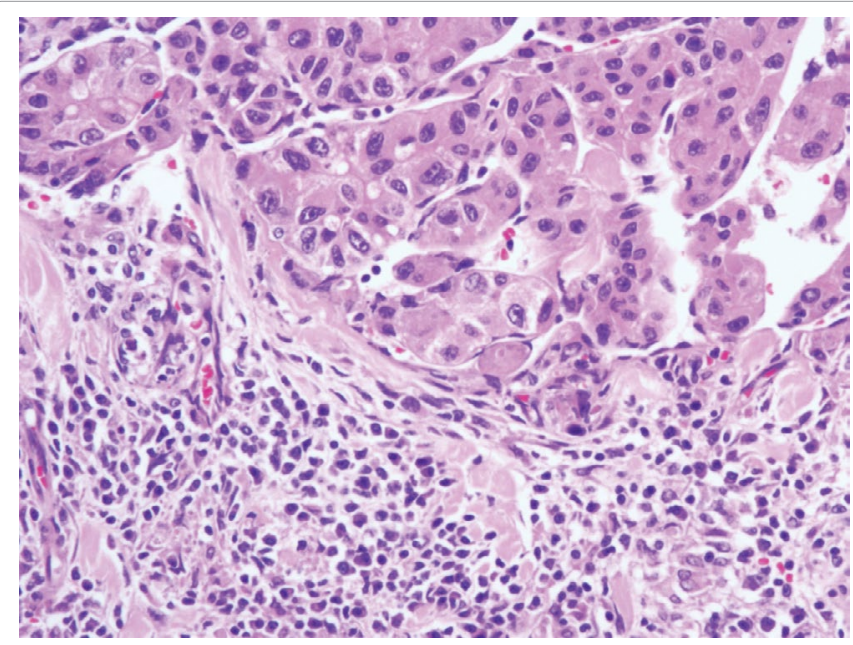

Figure 4: Enlarged right cervical lymph node: Cancer cells having oval and single nuclei in the lymph node; hematoxylin and eosin (HE) stain, 400x.

thrombus. The diagnosis was moderately differentiated HCC $\left(\mathrm{T}_{2} \mathrm{~N}_{0} \mathrm{M}_{0}\right.$, p Stage II UICC TNM classification). In October 2017, he was returned to our hospital because of right cervical lymph node swelling. Physical examination was unremarkable except for an abdominal surgical scar. The hematological and biochemical laboratory data were normal. Serum levels of DCP $(65 \mathrm{mAU} / \mathrm{ml})$ and AFP $(25.1 \mathrm{ng} / \mathrm{ml})$ were also normal. HBV DNA quantification was within normal limits. Abdominal ultrasonography, PET/CT, Contrast-enhanced CT did not show any tumors in the liver. However, Neck Ultrasound, PET/CT and Contrastenhanced MRI revealed right cervical lymph node swelling (about 23 $\times 39 \mathrm{~mm}$ ) (Figures 2 and 3). On the basis of laboratory, radiological and pathological data, he was diagnosed with HCC accompanied by metastasis of a right cervical lymph node. Thereupon, On November 1, 2017, He was treated with a right cervical lymphotomy, and the postoperative pathology confirmed this diagnosis (Figure 4).

\section{Discussion}

HCC is the seventh most common cancer in the world, and the third leading cause of cancer-related deaths. In China, it is also one of the most devastating malignancies. It causes more than 200,000 deaths annually and accounts for about 55\% of HCC deaths worldwide [5-8].

The distant metastasis of HCC usually occurs in three main ways: Hematogenous dissemination, lymphatic metastasis and implantation metastasis [9]. Lymph flow of the liver enters abdominal lymphatic system mainly through hepatoduodenal ligament. A small amount of flow communicates with supradiaphragmatic lymphatic system through the bilateral triangular ligaments. Katyal $S$ et al reported that sixty $(77 \%)$ of these 78 patients had involvement of the regional lymph nodes, and the remaining 18 (23\%) had distant lymph node involvement [2]. Only few case reports report cervical lymph node metastases of HCC [10-12]. Most HCC patients have underlying chronic liver disease and the carcinoma is usually accompanied by increased lymph production. The complex bypasses of lymphatic flow because of lymphatic occlusion may contribute to the occurrence of the skip metastasis of lymph nodes [13].

The incidence of lymph node metastasis in HCC patients was low, and patients with LNM had a poorer prognosis $[14,15]$. Sun H's study showed the 1-, 3-, and 5-year survival rates after surgery for HCC were $62.0 \%, 31.0 \%$, and $26.0 \%$ [16]. There is no standard treatment for extrahepatic metastases after hepatic resection of HCC. Lymphadenectomy, radiation therapy and Chemotherapy have been considered treatments for lymph node metastasis of HCC after hepatectomy [17-19]. Zeng's study identified 125 patients with HCC metastasis to regional LNs. The median survival for the external beam radiotherapy(EBRT) group was 9.4 months and the non-EBRT group was 3.3 months. They concludes EBRT is an effective palliative treatment for patients with LN metastases from HCC and may prolong overall survival [20]. Kobayashi S's study showed lymphadenectomy was a feasible and efficacious procedure to improve survival rates [21]. In our patient, the cervical lymph node was solitary, and there was no tumor in the residual liver or evidence of other extrahepatic metastases. We therefore resected the LN metastasis. Subsequently, we performed adjuvant chemotherapy with $400 \mathrm{mg}$ with twice daily of Sorafenib. However, cervical lymphadenopathy is not the common presentation in most conditions. In our case, when the patient was admitted to our hospital for the first time, although the physical examination did not detect the neck mass, we did not perform neck ultrasound or CT to verify this result. Therefore, at this point we can not assess whether patient had cervical lymph nodes.

\section{Conclusion}

In summary, the patients with LN metastasis from HCC have a poor Prognosis. And the metastasis of a cervical lymph node is rarely observed in patients with HCC. In the follow-up of patients with HCC, include who underwent surgical resection, should be considered the possibility of skip metastasis accompanying HCC. Therefore, overall physical examination, especially of the neck, should not be overlooked.

\section{References}

1. Sanyal AJ, Yoon SK, Lencioni R (2010) The etiology of hepatocellular carcinoma and consequences for treatment. Oncologist 15: 14-22.

2. Katyal S, Oliver JH, Peterson MS, Ferris JV, Carr BS, et al. (2000) Extrahepatic metastases of hepatocellular carcinoma. Radiology 216: 698-703.

3. Kim DY, Han K (2012) Epidemiology and surveillance of hepatocellular carcinoma. Liver Cancer 1: 2-14.

4. Yang JD, Roberts LR (2010) Epidemiology and management of hepatocellular carcinoma. Infect Dis Clin North Am 24: 899-919.

5. Watanabe J, Nakashima O, Kojiro M (1994) Clinicopathological study on lymph node metastasis of hepatocellular carcinoma: a retrospective study of 660 consecutive autopsy cases. Jpn J Clin Oncol 24: 37-41.

6. Yang BH, Xia JL, Huang LW, Tang ZY, Chen MS, et al. (2004) Changed clinica aspects of primary liver cancer in China during the past 30 years. Hepatobiliary Pancreat Dis Int 3: 194-198.

7. Yang JD, Roberts LR (2010) Hepatocellular carcinoma: A global view. Nat Rev Gastroenterol Hepatol 7: 448-458. 
Citation: Zhao Z, Maia J, Zhang X, Yan H, Chen X, et al. (2018) A Case Report on the Right Cervical Lymph Node Metastasis of Hepatocellular Carcinoma. J Cancer Sci Ther 10: 329-331. doi: 10.4172/1948-5956.1000563

8. Tang ZY (2002) Small hepatocellular carcinoma: Current status and prospects. Hepatobiliary Pancreat Dis Int 1: 349-353.

9. Liu T, Gao JF, Yi YX, Ding H, Liu W (2013) Misdiagnosis of left supraclavicula lymph node metastasis of hepatocellular carcinoma: A case report. World $\mathrm{J}$ Gastroenterol 19: 960-963.

10. Kobayashi K, Himoto T, Tani J, Miyoshi H, Yoneyama H, et al. (2012) A rare case of hepatocellular carcinoma accompanied by metastasis of a cervical lymph node. Intern Med 51: 381-385.

11. Fitzmorris PS, Morris JL, Fettig DM (2015) An unusual presentation of hepatocellular carcinoma. Am J Gastroenterol 110: 1395-1395.

12. Madabhavi I, Patel A, Choudhary M, Anand A, Panchal H, et al. (2014) Right cervical lymphadenopathy: a rare presentation of metastatic hepatocellular carcinoma. Gastroenterol Hepatol Bed Bench 7: 177-182.

13. Abe T, Furuse J, Yoshino M, Kinoshita T, Konishi M, et al. (2002) Clinical characteristics of hepatocellular carcinoma with an extensive lymph node metastasis at diagnosis. Am J Clin Oncol 25: 318-323.

14. Toyoda H, Fukuda Y, Koyama Y, Nishimura D, Hoshino H, et al. (1996) Case report: multiple systemic lymph node metastases from a small hepatocellular carcinoma. J Gastroenterol Hepatol 11: 959-962.

15. Uenishi T, Hirohashi K, Shuto T, Kubo S, Tanaka H, et al. (2000) The clinical significance of lymph node metastases in patients undergoing surgery for hepatocellular carcinoma. Surg Today 30: 892-895.

16. Sun HC, Zhuang PY, Qin LX, Ye QH, Wang L, et al. (2007) Incidence and prognostic values of lymph node metastasis in operable hepatocellular carcinoma and evaluation of routine complete lymphadenectomy. J Surg Oncol 96: $37-45$.

17. Liu CL, Fan ST (1997) Non-resectional therapies for hepatocellular carcinoma Am J Surg 173: 358-365.

18. Park YJ, Lim DH, Paik SW, Koh KC, Lee JH, et al. (2006) Radiation therapy for abdominal lymph node metastasis from hepatocellular carcinoma. J Gastroenterol 41: 1099-1106.

19. Ercolani G, Grazi GL, Ravaioli M, Grigioni WF, Cescon M, et al. (2004) The role of lymphadenectomy for liver tumors. Ann Surg 239: 202-209.

20. Zeng ZC, Tang ZY, Fan J, Qin LX, Ye SL, et al. (2005) Consideration of role of radiotherapy for lymph node metastases in patients with HCC: Retrospective analysis for prognostic factors from 125 patients. Int J Radiat Oncol Biol Phys 63: $1067-1076$

21. Kobayashi S, Takahashi S, Kato Y, Gotohda N, Nakagohri T, et al. (2011) Surgical treatment of lymph node metastases from hepatocellular carcinoma. J Hepatobiliary Pancreat Sci 18: 559-566. 